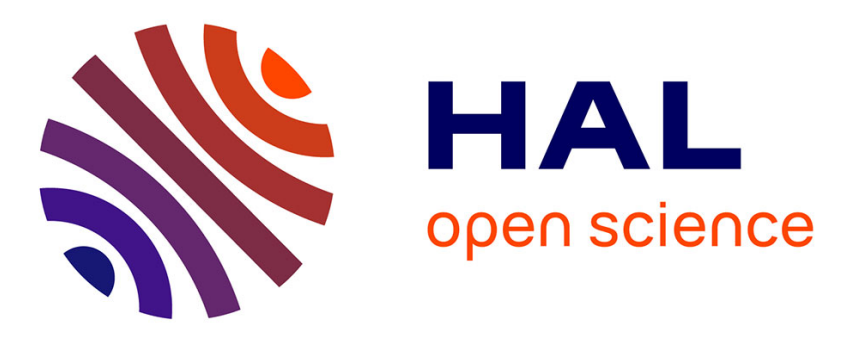

\title{
Macroscopic extension of RRK and Weisskopf models of unimolecular evaporation
}

Jean-Marc L'Hermite, Sébastien Zamith

\section{To cite this version:}

Jean-Marc L'Hermite, Sébastien Zamith. Macroscopic extension of RRK and Weisskopf models of unimolecular evaporation. EPL - Europhysics Letters, 2012, 100 (2), pp.23001. 10.1209/02955075/100/23001 . hal-00747636

\section{HAL Id: hal-00747636 \\ https://hal.science/hal-00747636}

Submitted on 31 Oct 2012

HAL is a multi-disciplinary open access archive for the deposit and dissemination of scientific research documents, whether they are published or not. The documents may come from teaching and research institutions in France or abroad, or from public or private research centers.
L'archive ouverte pluridisciplinaire HAL, est destinée au dépôt et à la diffusion de documents scientifiques de niveau recherche, publiés ou non, émanant des établissements d'enseignement et de recherche français ou étrangers, des laboratoires publics ou privés. 


\title{
Macroscopic extension of RRK and Weisskopf models of unimolecular evaporation
}

\author{
JEAN-MARC L'HERMITE ${ }^{1,2(a)}$ AND SEBASTIEN ZAMITH ${ }^{1,2}$ \\ ${ }^{I}$ Université de Toulouse ; UPS ; Laboratoire Collisions Agrégats Réactivité, IRSAMC ; F-31062 Toulouse, France \\ ${ }^{2}$ CNRS ; UMR 5589 ; F-31062 Toulouse, France
}

received and accepted dates provided by the publisher other relevant dates provided by the publisher

PACS 36.40.Qv - Stability and fragmentation of clusters

PACS 64.70.fm - Thermodynamics studies of evaporation and condensation

\begin{abstract}
The macroscopic behavior of two microscopic unimolecular evaporation models is examined. The temperature dependence of bulk equilibrium vapor pressures deduced from the RiceRamsperger-Kassel (RRK) model and from the Weisskopf model, respectively, are compared. The bulk vapor pressures of sodium given by both models are compared, over a wide temperature range, with the experimental vapor pressures. The Weisskopf model is in better agreement with the experimental data than the RRK theory: From the melting temperature to the critical temperature ( 370K-2500K), the vapor pressures of sodium calculated using the Weisskopf model agree with experimental values within $2 \%$, whereas RRK theory leads to errors of more than $40 \%$. Weisskopf theory satisfying detailed balance principle, whereas RRK theory does not, may explain this result.
\end{abstract}

\section{Introduction}

Evaporation is an ubiquitous physical phenomenon in which single particles escape from the solid or liquid state into the gas phase.

At the macroscopic scale, evaporated particles form a vapor, whose pressure depends on the nature of the substance and of its temperature. The evaporation rate of atoms or molecules from the bulk in solid or liquid state is characterized by its equilibrium vapor pressure, at which the evaporation probability exactly counterbalances the condensation probability. Many experimental methods exist since a long time for determining equilibrium vapor pressures of various substances [1]

At the molecular scale, thermal evaporation of atoms or molecules from single small objects, such as molecules or clusters, cannot be described, nor experimentally characterized, by an equilibrium vapor pressure, which is a statistical description of a large number of particles. However, the evaporation rate of isolated small particles can be experimentally investigated by mass spectrometry methods [2].

Some phenomena, such as droplets nucleation in a supersaturated vapor for instance, require a description of evaporation that should be valid at both microscopic and macroscopic scales. One way to obtain such a description is to extend asymptotically to infinite size systems the expressions for evaporation and condensation given by microscopic models. The macroscopic evaporation rates obtained must of course obey the known bulk properties; in particular, they must be able to reproduce the experimental evolution of equilibrium vapor pressures with temperature.

The present paper aims at establishing a bridge between microscopic models of thermal evaporation and the related properties of bulk. Experimental vapor pressures of sodium are compared here to calculated values obtained from the extension to macroscopic systems of two models widely used to describe microscopic evaporation: the RRK model and the Weisskopf model. Sodium is chosen as a typical example since the evaporation of sodium clusters is well documented and both RRK and Weisskopf models are able to reproduce microscopic experimental data [2].

A significant difference between Weisskopf and RRK approaches, which has important consequences as it will be shown, is that the RRK model does not account for the density of states of the evaporated particle (it is a non-microreversible model), whereas the Weisskopf model, which obeys the detailed balance principle (it is a microreversible model), does. It will be shown that this issue with RRK theory, which was already pointed out $[3,4,5]$, makes it incompatible with classical thermodynamics when applied to macroscopic systems.

The simple analytical expressions of RRK and Weisskopf evaporation rates of this paper are the most commonly used. The RRK expression of evaporation rates used here, in particular, is not one of the modified versions introduced by some authors to avoid its intrinsic violation of the detailed balance principle $[3,4]$. The purpose of the present study is not to obtain accurate macroscopic extension of RRK and 
Weisskopf theories, but to give a rationale for their macroscopic extension and to stress the failure of the nonmicroreversible RRK model, which is unable to reproduce some properties of macroscopic systems such as equilibrium vapor pressures. The evaporation rate given by RRK-based models is an Arrhenius law of the form $A \exp \left(-D / k_{B} T\right)$ where $A$ does not depend on the temperature, whereas the Weisskopf model gives an expression of the form $A / T \exp \left(-D / k_{B} T\right)$. Intermediate forms are also found in the literature, for instance $A / \sqrt{T} \exp \left(-D / k_{B} T\right)$ [6], Some authors, after having obtained the $1 / \mathrm{T}$ dependence of the prefactor in a microreversible scheme, finally give up this $1 / \mathrm{T}$ dependence and unjustifiably use a pure Arrhenius form with a constant prefactor [7],

A practical conclusion of the paper is that, when a simple analytical expression is required to describe evaporation, the second form $A / T \exp \left(-D / k_{B} T\right)$ is preferable, especially when dealing with multiscale phenomena that runs from microscopic to macroscopic scales.

\section{Microscopic models of unimolecular evaporation}

The calculation of equilibrium vapor pressure requires describing the two basic phenomena involved, the evaporation of atoms from the condensed phase and the reverse process, the attachment of atoms onto condensed phase systems. Equilibrium between the condensed phase and the vapor is reached when attachment and evaporation probabilities are equal: the fulfillment of this condition is used here to calculate the vapor pressure as a function of temperature.

Two simple analytical expressions for the macroscopic equilibrium vapor pressure of a monoatomic compound are derived below, starting from the RRK model and the Weisskopf model, respectively, to calculate evaporation rates and using the hard sphere approximation to evaluate attachment rate. Simple versions of RRK and Weisskopf models are used, which do not include anharmonicity nor rotation, where the condensed phase is described as a collection of harmonic oscillators at the same frequency and the vapor is considered as a perfect gas. This simplicity allows calculating analytical expressions of Weisskopf and RRK evaporation rates, and of classical thermodynamics properties. The main difference between RRK and Weisskopf models lies in the fact that Weisskopf approach accounts for the translational density of states of the evaporated atom or molecule, whereas RRK theory which is an "activated complex theory", does not. As a consequence, RRK-based theories are unable to account for the entropy of the macroscopic vapor.

We will first recall the basics of RRK and Weisskopf models, then we will derive analytical expressions of equilibrium vapor pressure as a function of temperature from both models and compare these expressions to experimental equilibrium vapor pressures of sodium.

The RRK evaporation rate
RRK model has been developed by Rice and Ramsperger [8] and, in parallel, by Kassel [9]. The basics of this well known theory are widely available in the literature [10]. Let's just recall the main ideas: the dissociation of the so-called "activated complex" occurs as soon as the necessary energy is accumulated in one of the vibrational modes that lead to dissociation, called "critical oscillators". The dissociation probability is calculated from the ratio of the total number of quantum states of all activated complexes relative to the total number of states accessible to the system.

A system of $n$ particles is represented as a collection of $s=3 n-6$ quantum harmonic oscillators of the same frequency $v$. The energy of each oscillator is $h v$. The system contains a total energy $E$, whose distribution among the oscillators fluctuates randomly at a frequency close to $v$. Evaporation occurs as soon as at least the dissociation energy $D$ is accumulated on one particular oscillator, in other words when at least $m>(D / h v)$ quanta are focused on this critical oscillator. Let us call $\mathrm{W}_{n>m}$ the total number of configurations that fulfill this condition, and $W_{n}$ the total number of configurations allowed for the system. The probability that a particular oscillator contains at least $m$ quanta is $P_{n>m}=W_{n>m} / W_{n}$ [10]. To obtain the probability that one atom evaporates from the whole cluster, this quantity is generally multiplied by the number of surface atoms $n_{e x t}[11,12,13]$. If one considers that the internal energy fluctuates at the frequency $v$, the evaporation probability per unit time is given by

$$
k_{\text {evap }}^{R R K}(n, E)=n_{\text {ext }} v(1-D / E)^{s-1}
$$

For large enough particles, the number of surface atoms of a spherical cluster containing $n$ atoms is $n_{\text {ext }} \approx(36 \pi)^{1 / 3} n^{2 / 3}$ $[11,12]$; in order to calibrate in the following the parameters $D$ and $v$ from the experimental bulk equilibrium vapor pressure, it is useful here to state the evaporation rate as a function of the hard sphere cross section $\sigma_{n}$. The radius $R$ of a cluster containing $n$ atoms scales as $r_{0} n^{1 / 3}$, and the hard sphere cross section $\sigma_{n}$ is equal to $\pi r_{0}{ }^{2} n^{2 / 3}$, thus $n_{\text {ext }}=(36 \pi)^{1 / 3} \sigma_{n} /\left(\pi r_{0}{ }^{2}\right)$. Expression (1) is valid in the microcanonical ensemble, at a given total energy $E$. However, in the canonical ensemble, as far as the activation energy $D$ is small compared with the mean total energy of the system $E$, the canonical rate constant $k_{\text {evap }}^{R R K}(n, T)$ is well approximated by the microcanonical rate constant $k_{\text {evap }}^{R R K}(n, \bar{E})$ [14]. The mean thermal internal energy is given here by $\bar{E}=s k_{B} T$. Finally, for large $n$, using $\lim _{n \rightarrow \infty}(1+x / n)^{n}=e^{x}$, the RRK evaporation rate constant is given by $[10,11,12]$

$k_{\text {evap }}^{R R K}(n, T)=\frac{(36 \pi)^{1 / 3} \sigma_{n}}{\pi r_{0}^{2}} v \exp \left(-\frac{D}{k_{B} T}\right)$

The Weisskopf evaporation rate 
An alternative description of thermal evaporation, initially developed to describe neutron evaporation from nuclei, was developed by V. Weisskopf [15]. There is an important conceptual difference between both theories: While the RRK model is based on properties of the density of states of the "activated complex" and does not account for the properties of the dissociated product (when the evaporated atom or molecule is infinitely separated from the cluster), the Weisskopf model explicitly considers the density of state of the evaporated subunit. The Weisskopf theory satisfies the detailed balance principle: The evaporation rate $W_{n}^{\text {evap }}$ and the attachment rate $W_{n-1}^{\text {stick }}$ must be linked by the following relation:

$$
\frac{W_{n}^{\text {evap }}\left(E_{n}, \varepsilon\right)}{W_{n-1}^{\text {stick }}}=\frac{\Omega_{n-1}\left(E_{n}-D_{n}-\varepsilon\right) \times \Omega_{\text {atom }}(\varepsilon)}{\Omega_{n}\left(E_{n}\right)}
$$

where $\varepsilon$ is the translationnal energy of the evaporated atom, $D_{n}$ is the dissociation energy (subscript $n$ refer here and in the following to the number of atoms in the cluster), $E_{n}$ is the total internal energy of the cluster, and $\Omega_{\mathrm{n}-1}, \Omega_{\text {atom } \square} \operatorname{and}_{\square} \Omega_{\mathrm{n}}$ are the density of states of the product cluster, the evaporated atom and the parent cluster, respectively. An analytic expression of the evaporation rate $W_{n}^{\text {evap }}$ can be obtained from relation (3) under the following approximations [4]: $\Omega_{\mathrm{n}}$ (and similarly $\Omega_{\mathrm{n}}$ ${ }_{1}$ ) is calculated assuming that, as in the RRK calculation presented above, clusters are represented by $s=3 n-6$ harmonic oscillators at the same frequency $v$, the density of states of the evaporated atom $\Omega_{\text {atom }}$ is assumed to be the translational density of states of a free particle of kinetic energy $\varepsilon$ in a volume $V, \Omega_{\text {atom }}=8 \pi \mu \varepsilon V /\left(h^{3} \mathrm{v}_{\text {atom }}\right)$, where $\mathrm{v}_{\text {atom }}$ is the velocity of the ejected atom and $\mu$ the reduced mass of the atom-cluster system; the attachment cross section is evaluated in the hard sphere approximation; rotational effects are neglected. Under these assumptions, the integration of relation (3) over all allowed energies $\varepsilon$ gives the following expression for the evaporation rate per unit time of a cluster of size $n$ containing an internal energy $E$ [4]:

$$
k_{n}^{\text {evap }}(n, E)=\sigma_{n-1} 8 \pi \mu v^{3}(s-1) \frac{\left(E-D_{n}\right)^{s-2}}{E^{s-1}} \text { (4) }
$$

where $\sigma_{n-1}$ is the attachment cross section of an atom onto the cluster of size $n-1$ and $D_{n}$ is the dissociation energy of a cluster containing $n$ atoms. For large enough clusters ( $D_{n} \rightarrow D_{\infty}=D, \mu \rightarrow m$ ), if the mean thermal internal energy is given by $\bar{E}=s k_{B} T$, and approximating again the canonical rate $k^{\text {evap }}(n, T)$ by the microcanonical rate $k^{\text {evap }}(n, \bar{E}(T))$ [14], equation (4) becomes:

$$
k_{\text {evap }}^{\text {Weissopf }}(n, T)=\sigma_{n-1} 8 \pi m v^{3} \frac{1}{k_{B} T} \exp \left(-\frac{D}{k_{B} T}\right)
$$

When applied to very small systems, expressions (1) and (4) are of course to be corrected to take into account the size variation of $D[2,16]$.

\section{The bulk equilibrium vapor pressure}

The equilibrium vapor pressure is calculated assuming that the number of evaporated atoms per unit time is the same as the number of atoms attached to the cluster during the same time. The attachment probability is deduced from the attachment cross section $\sigma_{n}$, calculated in the hard sphere approximation (thus independent of collision energy). The vapor of density $\rho$ is described as a perfect gas. The attachment rate is given by:

$$
k_{n}^{\text {stick }}(n)=\rho \sigma_{n} \mathrm{v}_{\text {atom }}
$$

where $\mathrm{v}_{\text {atom }}$ is the mean velocity of atoms. The bulk equilibrium vapor $P_{\text {sat }}$ is equal to $\rho_{s a t} k_{B} T$ in the perfect gas approximation. It is calculated here by setting $k_{n \rightarrow \infty}^{\text {stick }}=k_{n \rightarrow \infty}^{\text {evap }}(T) . k_{n \rightarrow \infty}^{\text {stick }}$ is given by relations (6) and $k_{n \rightarrow \infty}^{\text {evap }}(T)$ is given by relations (2) and (5), in the RRK and Weisskopf approximations, respectively. Finally, the equilibrium vapor pressure $P_{\text {sat }}^{R R K}$ and $P_{\text {sat }}^{\text {Weisskopf }}$ obtained from RRK and Weisskopf models, respectively, are given by the following relations:

$$
\begin{aligned}
& P_{\text {sat }}^{R R K}=\frac{(36 \pi)^{1 / 3}}{\pi r_{0}^{2}} \sqrt{\frac{\pi m k_{B}}{8}} \nu \exp \left(-\frac{D}{k_{B} T}+\frac{1}{2} \ln (T)\right) \\
& P_{\text {sat }}^{\text {Weiskopf }}=\frac{(2 \pi m)^{3 / 2} v^{3}}{\sqrt{k_{B}}} \exp \left(-\frac{D}{k_{B} T}-\frac{1}{2} \ln (T)\right)
\end{aligned}
$$

An expression similar to relation (8) is implicitly given by Bertsch and coworkers [17]. The most important difference between both expressions, beyond the difference in the prefactors (that are independent of temperature), is the temperature dependence of the last term in the exponential factor, equal to $+1 / 2 \ln (T)$ in the RRK expression and to $-1 / 2 \ln (T)$ in the Weisskopf expression. This difference in the temperature dependence of both models directly arises from the introduction of the translational density of states of the evaporated atoms in the Weisskopf model, thus it is closely related to the Weisskopf theory obeying detailed balance principle while RRK theory does not. Moreover, it is shown in appendix that the Weisskopf expression of $P_{\text {sat }}^{\text {Weiskof }}(T)$ is compatible with classical thermodynamics in the perfect gas approximation, whereas RRK expression of $P_{\text {sat }}^{R R K}(T)$ is not. 
Another difference between Weisskopf and RRK-derived expressions of $\mathrm{P}_{\text {sat }}$ lies in their different sensitivity to the energy dependence of the sticking cross section, which is not taken into account here. The equilibrium vapor pressure is less sensitive to the energy dependence of the sticking cross section when the Weisskopf model (or any microreversible model) is used, since the evaporation rate is "naturally" proportional to the sticking cross section, so that when writing the equilibrium condition (sticking rate $=$ evaporation rate), the cross section (and thus its possible energy dependence) vanishes within the approximations used in this paper. This might contribute to the almost surprisingly good agreement of Weisskopf expression of vapor pressures with experimental data. This argument does not hold in the RRK approach, where the sticking cross section is "artificially" introduced in the expression of evaporation rate (see relation (2)).

A widely used semi-empirical expression for vapor pressures is the Kirchhoff's expression $\ln P=-A / T-B \ln T+C$, where $A, B$ and $C$ are constant parameters that are tabulated. It is worth noting that $B$ is usually a positive constant (to very few exceptions such as helium) [18], which is consistent with expression (8) where $B=+1 / 2$, while it is in contradiction with expression (7) where $B=-1 / 2$.

\section{Comparison with experiment of calculated vapor pressure of sodium}

We take here the following Kirchhoff-type approximation of experimental vapor pressure of sodium $P_{\text {sat }}^{\exp }(T)$ [19]:

$\ln P_{\text {sat }}^{\exp }(T)=11.9463-12633 / T-0.4672 \ln T$

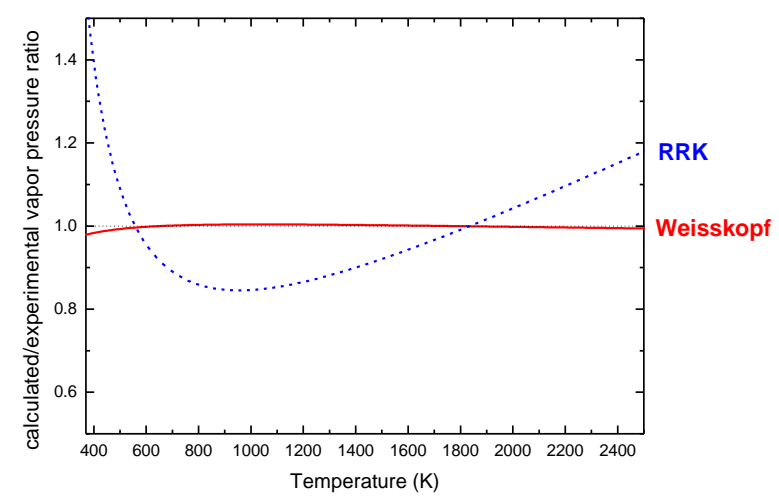

Fig. 1: (Colour on-line) ratio of calculated to experimental sodium vapor pressures. The (red online) solid line is obtained using Weisskopf model of evaporation and the (blue online) dashed line is obtained using RRK model of evaporation. The theoretical parameters $v$ and $D$ are determined from the best fit of experimental vapor pressures given by relation (9):

$$
\begin{aligned}
& v^{\text {Weisskopf }}=1.845 \times 10^{12} \mathrm{~s}^{-1} ; v^{R R K}=5.8 \times 10^{12} \mathrm{~s}^{-1} ; \\
& D^{\text {Weisskopf }}=1.0915 \mathrm{eV} ; D^{R R K}=1.009 \mathrm{eV}
\end{aligned}
$$

Figure 1 shows the ratio of the values given by expression (9) to the values calculated using relations (7) and (8), in the RRK and Weisskopf approximations, respectively. The comparison spans the whole temperature range from sodium melting temperature $(\sim 370 \mathrm{~K})$ up to its critical temperature $(\sim 2500 \mathrm{~K})$. The value of $r_{0}$ used in relation (7) is $2 \AA$ [16]. The two free parameters $v$ and $D$ are determined by a best fit approximation of expression (9). Using the Weisskopf approximation of $P_{\text {sat }}^{\exp }(T)$, the best fit parameters are $v=1.845 \times 10^{12} \mathrm{~s}^{-1}$ and $D=1.0915 \mathrm{eV}$; in the RRK approximation, the best fit values are $v=5.8 \times 10^{12} s^{-1}$ and $D=1.009 \mathrm{eV}$. These parameters are in agreement with expected values : the $D$ values are not far from the cohesive energy of bulk sodium $(D=1.13 \mathrm{eV} /$ atom [16]), and the $v$ values are compatible with the ones used in Weisskopf or RRK models to account for evaporation rates of free sodium clusters (the phonon frequency of the solid $v=3.1 \times 10^{12} s^{-1}$ is used in reference [2], and the value $v=2.35 \times 10^{12} s^{-1}$ is taken in reference [16]; the exact value of $V$ is not crucial in modelizing clusters evaporation rates). Figure 1 shows very clearly that the Weisskopf expression of vapor pressure given by relation (8) is in much better agreement with experiment that RRK's one given by relation (7). The discrepancy between the RRK curve and the experimental curve is much larger than the uncertainty on the approximation given by relation (9) of experimental data [19].

\section{Conclusion}

The calculation of bulk vapor pressure from RRK and Weisskopf models, respectively, has been exemplified here in the case of sodium, for which small clusters evaporation properties are well documented. It is shown that the bulk equilibrium vapor pressures of sodium calculated using Weisskopf model are in better agreement with experimental data than the ones obtained using RRK model. It is demonstrated that the violation of detailed balance principle by RRK theory makes it inconsistent with classical thermodynamics and is certainly responsible of the poor agreement with the experimental data. The free parameters of microscopic evaporation models, namely the vibrational frequency of the cluster $v$ and its dissociation energy $D$, can be deduced from experimental equilibrium vapor pressures. The values found for sodium using Weisskopf model, $D=1.09 \mathrm{eV}$ and $v=1.845 \times 10^{12} \mathrm{~s}^{-1}$, are very realistic. The method used here to deduce them from experimental equilibrium vapor pressures is a convenient way to calibrate them. The variation with temperature of the latent 
heat of evaporation given by classical thermodynamics is the same as the one deduced from Weisskopf theory, whereas it is not compatible with the one deduced from RRK theory (see appendix). Contrary to the RRK analytical expression of the bulk vapor pressure, the Weisskopf expression of the vapor pressure is compatible with the Kirchhoff expression for most compounds. Finally, it is shown that, when a simple expression is needed, an expression of the form $A / T \exp \left(-B / k_{B} T\right)$ seems to be preferable to an expression of the form $A \exp \left(-B / k_{B} T\right)$ (where $A$ and $B$ do not depend on temperature in both cases) to evaluate the evaporation probability of a substance, especially when multiscale reliability from microscopic scale to macroscopic scale is required.

\section{Appendix}

The temperature dependence of the latent heat of evaporation $L(T)$ can be calculated from Weisskopf and RRK evaporation rate and using the Clausius-Clapeyron relation. It is shown below that $L^{\text {Weisskopf }}(T)$ deduced from the Weisskopf expression of evaporation rate is in agreement with classical thermodynamics (at the same approximation level as in the Weisskopf model concerning the description of the cluster and the vapor), whereas $L^{R R K}(T)$ is not.

Considering the vapor as a perfect gas and neglecting the variation of volume of the condensed phase, the ClausiusClapeyron gives the following relation between the latent heat of evaporation $L$ and the temperature dependence of equilibrium vapor pressure $P_{\text {vap }}$ :

$$
L=k_{B} T^{2} \frac{d \ln P_{v a p}}{d T}
$$

Using this relation, the expression of $L^{\text {Weisskopf }}$ and $L^{R R K}$ can be deduced from relations (7) and (8), respectively:

$$
\begin{aligned}
& L^{\text {Weisskopf }}(T)=D-1 / 2 k_{B} T \\
& L^{R R K}(T)=D+1 / 2 k_{B} T
\end{aligned}
$$

Classical thermodynamics, in the perfect gas approximation, gives the following temperature dependence of the latent heat:

$$
L^{\text {classical }}(T)=L(0)+\left(C_{p}^{\text {vapor }}-C_{p}^{\text {cluster }}\right) T
$$

which is easily calculated for a cluster-perfect gas system from the following relations:

$$
\begin{aligned}
& L(0)=D \\
& \bar{E}_{\text {cluster }}=(3 n-6) k_{B} T \Rightarrow C_{p}^{\text {cluster }} \approx C_{v}^{\text {cluster }} \approx 3 k_{B} \\
& C_{p}^{\text {vapor }}=C_{v}^{\text {vapor }}+k_{B}=5 / 2 k_{B} \\
& \text { Finally, } L^{\text {classical }}(T)=D-1 / 2 k_{B} T, \text { which is the }
\end{aligned}
$$
same expression as $L^{\text {Weisskopf }}(T)$ given above by relation (b).

\begin{abstract}
$* * *$
We gratefully acknowledge Pierre Labastie for stimulating discussions and thorough advices.
\end{abstract}

\section{REFERENCES}

[1] For monoatomic vapors, see for instance R.W. Ditchburn And J.C. Gilmour, Rev. Mod. Phys. 12 (1941) 310

[2] C. BrÉChignac, Ph. CAhuZAC, J. LEYGNiER, AND J. Weiner, J. Chem. Phys. 90 (1989) 1492

[3] P. C. Engelking, J. Chem. Phys. 85 (1986) 3103

[4] Paul C. Engelking, J. Chem. Phys. 87 (1987) 936

[5] S.H. LIN, K.H. LAU, W.RICHARDSON, L.VOLK, AND

H. EYrIng, Proc. Nat. Acad. Sci. USA 69 (1972) 2778

[6] J. Borggreen, F. Chandezon, O. Echt, H. Grimley, K. HANSEN, P.M. HANSEN, AND C. Ristori, Eur. Phys. J. D 9 (1999) 119

[7] ROMAN RABINOVITCH, KlaVs HaNSEN, AND Vitaly V. Kresin, J. Phys. Chem. A 115 (2011) 6961

[8] O.K. RiCE AND H.C. RAMSPERger, J. Am. Chem. Soc. 49 (1927) 1617

[9] L.S. KASSEL, J. Phys. Chem. 32 (1928) 225

[10] See for instance WENDELl FORST, Unimolecular Reactions, a concise introduction (Cambridge University Press 2003), M.F. JARROLD, Introduction to statistical reaction rates theories, in Clusters of Atoms and Molecules I, editor H. HABERLAND, (Springer, Berlin, 1994), or R.D. LEVINE, Molecular Reaction Dynamics (Cambridge University Press 2005)

[11] MiCHAEL I. ZEIFMAN, BARBARA J. GARRISON, AND LeOnid V. ZhigIlei, J. Appl. Phys. 92 (2002) 2181

[12] KARINE GOURIET, MARC SENTIS, AND TATIANA E. ItINA, J. Phys. Chem. C 113 (2009) 18462

[13] C. BRÉChIGNAC, H. Busch, PH. CAHUZAC, AND J. LeYgnier, J. Chem. Phys. 101 (1994) 6992

[14] Cornelius E. Klots, J. Chem. Phys. 83 (1985) 5854

[15] V. WeISSKOPf, Phys. Rev. 52 (1937) 295

[16] P.A. Hervieux, D.H.E. Gross, Z. Phys. D 33 (1995) 295

[17] G.F. BERTSCh, N. OBERHOFER, AND S. STRINGARI, Z. Phys. D - Atoms, Molecules and Clusters 20 (1991) 123); our expression (8) is obtained in this paper by injecting relation (7) into relation (4).

[18] R.W. Ditchburn And J.C. Gilmour, Rev. Mod. Phys. 13 (1941) 310

[19] J.K. FINK, L. LEIBOWITZ, Thermodynamic and transport properties of sodium liquid and vapor, Report ANL/RE-95/2, Reactor Engineering Division, Argonne National Laboratory (1995) 235; DOI 10.2172/94649 